\title{
Hetero-resistance: an under-recognised confounder in diagnosis and therapy?
}

\begin{abstract}
There is no formally accepted definition for the term 'hetero-resistance', but in its broadest sense it may be understood as mixed populations of drug-resistant and drug-sensitive organisms in a single clinical specimen or isolate. However, it is certainly sensible to require additionally that the proportion of resistant organisms may not be explicable by the natural 'background' mutation rate alone. In a most stringent sense, not used here, one might even request that hetero-resistance is an exclusively phenotypic manifestation within a genetically homogeneous strain.
\end{abstract}

The number of species for which hetero-resistance has been described is very small but whenever heteroresistance is present it is almost certain to cause diagnostic or therapeutic problems, or both.

\section{Hetero-resistance in Staphylococcus aureus}

The species in which hetero-resistance has been reported most often and where its mechanisms are understood best is Staphylococcus aureus. In this organism, hetero-resistance to methicillin is dependent on the structural gene mecA which codes for a lowaffinity penicillin-binding protein (PBP2'). Expression of this protein causes strain-specific, variable resistance from borderline to elevated basal resistance to methicillin and all other $\beta$-lactam antibiotics. It can be induced by increased osmolarity, lowered temperature and other factors [1].

In addition to this low-level resistance, $m e c A$-positive $S$. aureus (MRSA) possess an intrinsic ability to generate a small proportion $\left(10^{-8}-10^{-4}\right.$ [2]) of highly resistant subclones. The genes responsible for this conversion to high-level resistance have not yet been identified, but it has been shown that the conversion must be due to chromosomal mutation(s) that are not related to $\operatorname{mec} A[1,2]$.

In contrast, vancomycin (intermediate) resistance is still extremely rare in $S$. aureus, but hetero-resistance to this drug has already been reported in as many as $9 \%$ of 129 MRSA isolates in a survey of seven Japanese university hospitals [3], in a case report from the UK [4], and in three MRSA cases from Hong Kong [5]. Recently, hetero-resistance has also been reported for teicoplanin [6]. The basis for vancomycin and teico- planin hetero-resistance is still unknown. It has been suggested that previously reported vancomycin heteroresistant $S$. aureus merely represent one end of a spectrum of low-level vancomycin resistance expression [7]. However, heterogeneous populations from which subclones could be generated with a vancomycin MIC of up to $16 \mathrm{mg} / \mathrm{L}$ for $S$. aureus and up to $64 \mathrm{mg} / \mathrm{L}$ for coagulase-negative staphylococci have been reported [5].

Undoubtedly, hetero-resistance in S. aureus leads to diagnostic problems. It has been shown that heteroresistant MRSA are easily overlooked or misidentified as borderline oxacillin-resistant $S$. aureus [8]. Similarly, vancomycin hetero-resistant staphylococci are easily overlooked. The disk diffusion test and several automated methods of susceptibility testing failed to detect glycopeptide-intermediate, possibly hetero-resistant $S$. aureus [9]. As vancomycin hetero-resistance appears to be inducible by salt and probably by $\beta$-lactam antibiotics [5], a screening method was proposed that looked for satellitism on Mueller-Hinton agar supplemented with vancomycin $4 \mathrm{mg} / \mathrm{L}$ and $\mathrm{NaCl}$ $4 \%$, around an aztreonam disk, which, in contrast to other $\beta$-lactam antibiotics, has no inhibitory effect on gram-positive bacteria [5].

It is not surprising that due to the diagnostic problems, hetero-resistance, especially to vancomycin, has repeatedly been suggested as an explanation for at least some of the therapeutic failures seen in infections with vancomycin-susceptible MRSA [3]. Furthermore, because of the inducibility of vancomycin heteroresistance by $\beta$-lactams, the typical clinical sequence of starting therapy with $\beta$-lactams, failure to improve clinically or detection of MRSA, and continuing with vancomycin has been implicated in the generation of vancomycin hetero-resistance [5].

\section{Hetero-resistance in bacteria other than S. aureus}

Besides S. aureus, hetero-resistance to methicillin or vancomycin, or both, has also been described for coagulase-negative staphylococci, including $S$. epidermidis, S. capitis, S. haemolyticus, S. auricularis, $S$. simulans and $S$. warneri $[5,10]$. Reports on heteroresistance in genera other than staphylococci are rare. 
Examples are metronidazole hetero-resistance in Gardnerella vaginalis, blamed for therapeutic failures [11], and hetero-resistance to fluconazole and other azoles in Cryptococcus neoformans [12]. As in the case of staphylococci, the latter type of resistance was shown to be innate. Resistant subcultures could be generated but purely sensitive subcultures could not, and resistant subcultures lost their resistance and returned to the stable initial hetero-resistant phenotype after maintenance on drug-free media [12].

\section{Hetero-resistance in mycobacteria is different}

Research on hetero-resistance in mycobacteria is still in its infancy but the resistance mechanisms themselves are well understood. In Mycobacterium tuberculosis, the vast majority of resistances are caused by point mutations in a limited number of genes whose products either are the target of or activate the antituberculosis drug. These are typically one-step events immediately leading to a high level of resistance.

Interestingly, $M$. tuberculosis susceptibility testing is unique in that a small number of resistant colonies is often seen and tolerated. For example, the widely accepted and WHO endorsed ' $1 \%$ proportion method' [13] allows for up to $1 \%$ resistant organisms in the primary isolate before the result of the susceptibility testing is declared to be resistant. This proportion is well above the spontaneous mutation rate, which is, for example, in the order of $c \cdot 10^{-8}$ for rifampicin and isoniazid. Although based on empiricism rather than scientific rationale, there is a general consensus that the clinical outcome in such cases correlates more with infections with uniformly sensitive organisms than with purely resistant populations [13].

Another finding not explicable by spontaneous mutation rates alone arises from the results of a recent molecular investigation of $54 \mathrm{M}$. tuberculosis DNA samples isolated directly from the sputum of patients with risk factors for resistance development, such as prior anti-tuberculosis treatment. Nine of these samples $(17 \%)$ revealed mixed populations of organisms with or without resistance-causing mutations. Interestingly, most of them ( 7 of 9) were reported as sensitive after conventional culture-based susceptibility testing, although they contained as many as $50 \%$ organisms with resistance-causing mutations, as estimated from cloning and sequencing products from independent PCR amplifications run in parallel on the same sample [14].

\section{Hetero-resistance in M. tuberculosis is well hidden}

If mixed populations are indeed as frequent in $M$. tuberculosis as these molecular investigations suggest, why are they not seen more often? The answer may come from studies on the relative biological fitness of
M. tuberculosis organisms with and without resistancecausing mutations. It was shown that acquisition of resistance results in an (at least temporary) decrease in growth rate $[15,16]$. Indeed, all currently employed routine techniques for phenotypic resistance testing and most available molecular resistance prediction tests depend on an initial, drug-free primary culture. It is not difficult to imagine that the wild-type organisms will tend to outgrow the biologically less fit mutants. And if this was not enough, nobody knows whether the decontamination step carried out before starting the culture might affect the hetero-resistant populations differently.

To further obscure the presence of hetero-resistant populations, it has been shown that even the two most widely used molecular techniques for mutation detection, PCR followed by direct sequencing or singlestrand conformation polymorphism, which could theoretically be applied directly to sputum samples independently of a primary culture, have been shown to be inefficient for detecting mixed populations [14, 17]. Although this problem may be resolvable by techniques such as restriction fragment-length polymorphism or the 'line probe assay', there is a further confounder affecting all culture-independent molecular resistance prediction tests because they all rely on an initial DNA amplification step. It has been shown that a mixture of templates, which is characteristic for DNA isolations from hetero-resistant populations, can result in a wide spectrum of PCR products, ranging from almost purely wild-type products, through various proportions of mixed products, to almost only the mutant genotype [14] - a phenomenon attributed to stochastic events during the early PCR cycles. It is clear that if this observation proves to be a common phenomenon there would be a need for far more than a single PCR run and subsequent product characterisations, imposing serious financial and practical limitations on the applicability of such techniques.

\section{Room for speculation}

Apart from implications for molecular resistance prediction tests, the apparent frequency of undetected hetero-resistance also offers attractive and rather simple explanations for peculiarities inherent to the diagnosis and treatment of tuberculosis. The ' $1 \%$ proportion method' has already been mentioned. A second example might be the empirically proven recommendation to use up to four different antituberculosis drugs for the treatment of tuberculosis, even when no resistances are suspected. Such a regimen would be quite unusual for the treatment of most other noncomplicated, bacterial infections. Certainly, this cannot be explained away entirely by unusual features of mycobacteria, such as a narrow therapeutic range and synergic effects of antituberculosis drugs, because this is also true for a number of other antibiotics, nor by 
their isolation inside cavities, as other bacteria can be sequestered, e.g., inside abscesses.

Further questions remain unresolved at this time. Of central importance is whether hetero-resistance in $M$. tuberculosis is caused by infections with two strains differing in their resistance pattern, or if the resistant subpopulation is clonal and develops during the course of infection. Studies are currently in progress to isolate homogeneous resistant and sensitive 'pairs' of strains originating from the same, hetero-resistant clinical sample to answer this question by fingerprint analysis.

Finally, it will be very interesting to find out if heteroresistance affects the clinical prognosis of patients with tuberculosis. This question could be answered by casecontrol studies with cases and controls matched for their phenotypic resistance pattern. It would be particularly useful to find out if hetero-resistance influences the frequency of 'developing' a resistance under therapy. If this knowledge were available, the affected patient could then benefit from an early adaptation of the therapeutic regimen. In this way, detection of hetero-resistance, at least in tuberculosis, may have more of a beneficial aspect rather than just leading to diagnostic confusion.

HEINZ RINDER

Department of Tropical Medicine and Infectious Diseases University of Munich 80802 Munich, Germany

I thank Dr Petras Stackenas, Vilnius, Professor Knut Feldmann, Gauting, Professor Jürgen Heesemann, Munich, and Professor Thomas Löscher, Munich, for constructive criticism.

\section{References}

1. Ryffel C, Strässle A, Kayser FH, Berger-Bächi B. Mechanisms of heteroresistance in methicillin-resistant Staphylococcus aureus. Antimicrob Agents Chemother 1994; 38: 724-728.

2. Strandén AM, Roos M, Berger-Bächi B. Glutamine synthetase and heteroresistance in methicillin-resistant Staphylococcus aureus. Microb Drug Resist 1996; 2: 201-207.

3. Hiramatsu K, Aritaka N, Hanaki H et al. Dissemination in Japanese hospitals of strains of Staphylococcus aureus heterogeneously resistant to vancomycin. Lancet 1997; 350: $1670-1673$.

4. Howe RA, Bowker KE, Walsh TR, Feest TG, MacGowan AP. Vancomycin-resistant Staphylococcus aureus (letter). Lancet 1998; 351: 602.

5. Wong SSY, Ho PL, Woo PCY, Yuen KY. Bacteremia caused by staphylococci with inducible vancomycin heteroresistance. Clin Infect Dis 1999; 29: 760-767.

6. Park YJ, Kim M, Oh EJ, Lee SO, Kim BK, Kim SM. Screening method for detecting staphylococci with reduced susceptibility to teicoplanin. J Microbiol Methods 2000; 40: 193-198.

7. Howe RA, Wootton M, Walsh TR, Bennett PM, MacGowan AP. Expression and detection of hetero-vancomycin resistance in Staphylococcus aureus. J Antimicrob Chemother 1999; 44: 675-678.

8. Liu H, Buescher G, Lewis N, Snyder S, Jungkind D. Detection of borderline oxacillin-resistant Staphylococcus aureus and differentiation from methicillin-resistant strains. Eur J Clin Microbiol Infect Dis 1990; 9: 717-724.

9. Tenover FC. Implications of vancomycin-resistant Staphylococcus aureus. J Hosp Infect 1999; 43 Suppl: S3-S7.

10. Cimolai N, Trombley C, Zaher A. Oxacillin susceptibility of coagulase-negative staphylococci: role of mecA genotyping and E-test susceptibility testing. Int J Antimicrob Agents 1997; 8: $121-125$.

11. Altrichter T, Heizmann WR. Gardnerella vaginalis: Transport, Mikroskopie, Resistenztestung. [Gardnerella vaginalis: transport, microscopy, testing resistance.] Geburtsh Frauenheilk 1994; 54: 606-611.

12. Mondon P, Petter R, Amalfitano G et al. Heteroresistance to fluconazole and voriconazole in Cryptococcus neoformans. Antimicrob Agents Chemother 1999; 43: 1856-1861.

13. Canetti G, Fox W, Khomenko A et al. Advances in techniques of testing mycobacterial drug sensitivity, and the use of sensitivity tests in tuberculosis control programmes. Bull World Health Organ 1969; 41: 21-43.

14. Rinder H, Mieskes KT, Löscher T. Heteroresistance in Mycobacterium tuberculosis. Int J Tuberc Lung Dis 2001; 5: 339-345.

15. Billington OJ, McHugh TD, Gillespie SH. Physiological cost of rifampin resistance induced in vitro in Mycobacterium tuberculosis. Antimicrob Agents Chemother 1999; 43: 1866-1869.

16. Gillespie SH. Antibiotic resistance in the absence of selective pressure. Int J Antimicrob Agents 2001; 17: 171-176.

17. Cooksey RC, Morlock GP, Holloway BP et al. Comparison of two nonradioactive, single-strand conformation polymorphism electrophoretic methods for identification of $r p o B$ mutations in rifampin-resistant isolates of Mycobacterium tuberculosis. Mol Diagn 1998; 3: 73-80. 